\title{
A prospective comparison study of early functional outcomes after implant-based breast reconstruction: subpectoral versus prepectoral technique
}

\author{
Joon Seok Lee ${ }^{1 \#}$, Eunhee Park ${ }^{2 \#}$, Jung Ho Lee ${ }^{1}$, Jeeyeon Lee ${ }^{3}$, Ho Yong Park ${ }^{3}$, Jung Dug Yang ${ }^{1}$, \\ Tae-Du Jung ${ }^{2}$
}

${ }^{1}$ Department of Plastic and Reconstructive Surgery, School of Medicine, Kyungpook National University, Daegu, Korea; ${ }^{2}$ Department of Rehabilitation Medicine, School of Medicine, Kyungpook National University, Daegu, Korea; ${ }^{3}$ Department of Surgery, School of Medicine, Kyungpook National University, Daegu, Korea

Contributions: (I) Conception and design: JS Lee, E Park; (II) Administrative support: J Lee, HY Park; (III) Provision of study material or patients: JS Lee, E Park, JH Lee (IV) Collection and assembly of data: JS Lee, E Park, JH Lee, J Lee; (V) Data analysis and interpretation: JS Lee, E Park, JD Yang, TD Jung; (VI) Manuscript writing: All authors; (VII) Final approval of manuscript: All authors.

\#These authors contributed equally to this work as co-first authors.

Correspondence to: Tae-Du Jung, MD, PhD. Department of Rehabilitation Medicine, School of Medicine, Kyungpook National University, 130 Dongduk-ro, Daegu, 41944, Korea. Email: teeed0522@knu.ac.kr; Jung Dug Yang, MD, PhD. Department of Plastic and Reconstructive Surgery, School of Medicine, Kyungpook National University, 130 Dongduk-ro, Daegu, 41944, Korea. Email: lambyang@knu.ac.kr.

Background: After skin-sparing mastectomy, direct-to-implant breast reconstruction is divided into a subpectoral and prepectoral techniques. However, there is still lack of studies that have compared the functional recovery after reconstruction based on the two techniques. Therefore, this study was conducted as a prospective comparison of the functional recovery and quality of life between the two techniques.

Methods: Patients who had undergone mastectomy for breast cancer were grouped based on whether the approach during direct-to-implant reconstruction was subpectoral or prepectoral. Functional outcomes were evaluated pre-operatively, 2 weeks, 1, 3, and 6 months post-operation. The evaluation included range of motion of the shoulder, maximal muscle power of the shoulder, pain intensity (measured by the visual analogue scale), disability of the upper extremity (measured by the Disabilities of the Arm, Shoulder and Hand questionnaire), quality of life (measured by the 36-Item Short-Form Health Survey), and mood status [measured by the hospital anxiety and depression scale (HADS)]. Repeated-measures analysis of variance was performed to evaluate changes in functional assessments.

Results: In the subpectoral/prepectoral groups, the average age, body mass index (BMI), preoperative breast volume, and implant volume were $45.5 / 45.1$ years, $22.6 / 22.7 \mathrm{~kg} / \mathrm{m}^{2}, 244.5 / 206.0 \mathrm{~cm}^{3}$, and $258.6 /$ $234.8 \mathrm{~cm}^{3}$, respectively. There were no significant differences in functional assessments between the two groups before the operation. There were significant differences in visual analogue scale, Disabilities of the Arm, Shoulder and Hand (DASH) scores, and time interaction effects between the two groups. In post-hoc analysis, the prepectoral group exhibited lower visual analogue scale and DASH scores than those in the subpectoral group at 2 weeks post-operation.

Conclusions: The prepectoral group showed favorable recovery of pain intensity and disability of the upper arm at the early phase post-operation. Both groups functionally recovered at 6 months post-operation. Therefore, the prepectoral technique can be considered as a useful alternative technique, compared to the classic subpectoral technique.

Keywords: Direct-to-implant breast reconstruction; pre-pectoral technique; sub-pectoral technique; early functional outcomes 
Submitted Aug 05, 2020. Accepted for publication Nov 27, 2020.

doi: 10.21037/apm-20-1550

View this article at: http://dx.doi.org/10.21037/apm-20-1550

\section{Introduction}

Due to its oncological safety, immediate breast reconstruction is considered indispensable in the surgical management of breast cancer patients. In recent years, a variety of bioprosthetic or synthetic materials have been developed, and various techniques have been devised and developed using a powerful tool called the acellular dermal matrix $(\mathrm{ADM})$; therefore, prepectoral implant breast reconstruction is gaining attention. Previously, the most commonly used technique was a subpectoral dual plane technique. While this technique has been used in the past to minimize numerous complications and has been highly valued for cosmetic satisfaction, there are reports that the placement of the insertion implant can cause postoperative pain and prolonged functional recovery (1-3). Recently, prepectoral direct-toimplant (DTI) breast reconstruction has been reported as a new paradigm. The greatest difference of this technique is that the silicone implant is wrapped in a variety of designs and inserted in the breast parenchyma without touching the pectoralis major muscle. This method has the advantage of reinforcing the contact part of the skin flap and implant. Moreover, there are a few cosmetically satisfying outcomes for the various wrapping design techniques (4-6). However, comparative studies on the rise in quality of life (QOL) due to functional and rapid recovery, by preserving the pectoralis major muscle, are still insufficient. The functionality, pain, and disability during recovery, and the recovery time course are concerns that surgeons performing breast reconstruction should be aware of.

The pectoralis major muscle is located under (downside) the breast and plays a role in the flexion, adduction, and internal rotation of the humerus. It is attached to the anterior surface of the sternum and is connected to the 6th or 7th rib cartilage with low down. During DTI breast reconstruction, the pocket is covered with $\mathrm{ADM}$ after the insertion of the implant by partially cutting the attachment of the inferolateral area while undermining the subpectoral plane. This procedure itself is likely to increase the possibility of pain because of the primary dissection and separation of the muscles, which involve pushing of the foreign body silicone implant in a direction different from the original anatomical position. Prolonged pain may lead to poor QOL, patient dissatisfaction, and physical and mental concerns.

Compared to breast reconstruction using autologous tissue, DTI breast reconstruction leads to a relatively faster recovery and an easier return to daily life, and there is no donor site morbidity. DTI is also believed to be a useful breast reconstruction method following mastectomy for patients who have already undergone augmentation mammoplasty.

The concept of prepectoral subcutaneous implant breast reconstruction was introduced in the 1970s; however, implant exposure due to skin flap necrosis and numerous other complications occurred. Therefore, DTI breast reconstruction technique that employs a subpectoral dual plane concept, using various methods and materials, was developed (4,7-9). This technique aims to minimize potential implant displacement, malposition, and pain by distributing the force exerted by the partial detachment of the costal arch and sternum to the infero-lateral part of the pectoralis major muscle. However, it has been reported that this technique may cause animation deformity of the pectoralis major muscle, pain, implant dislocation, or malposition.

Recently, a variety of techniques for implant coverage using ADM and prepectoral DTI breast reconstruction, which preserves the pectoralis major muscle from the start, have shown favorable results (4-6). By preserving the pectoralis major muscle, pain was reduced and the risk of animation deformity was minimized. However, when the thickness of the skin flap is thin, complications such as rippling deformity or depression of the implant boundary may occur. Schaeffer et al. reported early functional outcomes of prepectoral breast reconstruction (10), and Alba $e t a l$. analyzed postoperative upper extremity function after implant and autologous breast reconstruction (11). However, there is paucity of reports comparing the postoperative functional recovery based on whether the subpectoral and prepectoral techniques was employed.

The aim of this prospective study was to compare early functional recovery and QOL in a group of patients who underwent immediate DTI breast reconstruction after mastectomy using a classical subpectoral dual plane technique to insert the implant below the pectoralis major muscle.

We present the following article in accordance with the 
STROBE reporting checklist (available at http://dx.doi. org/10.21037/apm-20-1550).

\section{Methods}

\section{Study design}

The study was conducted in accordance with the Declaration of Helsinki (as revised in 2013). The Institutional Review Board of Kyungpook National University Chilgok Hospital (No. 2018-05-005-001) approved this prospective study, and all patients provided informed consent to have their data (including de-identified photographs) recorded, analyzed, and published for research purposes.

From February 2018 to September 2019, 34 patients who had skin-sparing mastectomy for breast cancer underwent DTI breast reconstruction using the subpectoral approach [subpectoral technique group, $\mathrm{n}=14$ (14 breasts)] or the prepectoral approach [prepectoral technique group; $\mathrm{n}$ $=20$ patients $(20$ breasts $)]$. The following patient characteristics were evaluated: age, body mass index (BMI), preoperative breast volume, implant volume, cancer staging, smoker/non-smoker, radiotherapy, and chemotherapy.

The functional outcome assessments were performed pre-operatively (T0), and at 2 weeks (T1), 1 month (T2), 3 months (T3), and 6 months post-operation (T4). The assessment involved range of motion of the shoulder, maximal muscle power of the shoulder, pain intensity, disability of the upper extremity, QOL, and mood status.

\section{Patient selection}

This prospective randomized study included patients: (I) who were diagnosed with breast cancer, (II) who immediately received breast reconstruction using implants during a breast cancer operation, and (III) who were 3060 years old at the time of the operation.

This study excluded patients: (I) who were diagnosed with advanced-stage III or IV breast cancer, (II) who were not able to complete the self-reported questionnaire due to a history of cognitive impairment, (III) who had a history of any neurologic and musculoskeletal disorder, and (IV) who had a history of alcohol or drug abuse. Prepectoral DTI breast reconstruction patients generally have a low risk of skin invasion, a small-to-moderate size of the reconstructed breast, and an adequate skin flap (thickness and vascularity), following mastectomy. To minimize the risk of flap loss, we only selected patients with a skin thickness $\geq 1.5 \mathrm{~cm}$ on the preoperative pinch test and a uniform thickness of $\geq 0.5 \mathrm{~cm}$ for the subcutaneous layer in the skin flap, post-mastectomy. A severely ptotic shape of the affected breast and obesity $\left(\mathrm{BMI}>30 \mathrm{~kg} / \mathrm{m}^{2}\right)$, diabetes mellitus, active smoking, and preoperative radiotherapy were considered as exclusions. When the inclusion criteria were sub-optimally met, classical subpectoral breast reconstruction was planned, and patients were divided into two groups for comparison.

\section{DTI breast reconstruction operative technique}

\section{Classic subpectoral DTI breast reconstruction}

The appropriate implant was selected based on the breast width and volume, and the excised mass weight was measured preoperatively. Undermining of the pectoralis major muscle was carefully performed to create a pocket that minimized bleeding and damage to the surrounding tissues. For insufficient muscle coverage in the infero-lateral region, an implant pocket was created using $\mathrm{ADM}$ (Figure 1).

\section{Prepectoral DTI breast reconstruction}

After selecting the appropriate implant for the patient, the two double-crossed ADMs technique, which is a technique with full coverage of the silicone implant, was applied for insertion of implant into the pocket (Figure 2) (6).

In both groups, prior to the insertion of the implant into the pocket, two lines with negative suction were applied to the breast pocket (in the upper part and in the inflammatory fold), and irrigation was performed using antibiotic irrigation solutions (povidone-iodine, $50 \mathrm{cc}$; isepamicin, $80 \mathrm{mg}$; cefazolin, $1 \mathrm{~g}$ in $500 \mathrm{~mL}$ of sterile saline). After betadine re-draping, the implant was positioned and an absorbable suture was placed to minimize future malpositioning.

\section{Functional outcome measurement}

\section{Intensity of pain}

The visual analog scale (VAS) was used to assess the pain intensity during the shoulder abduction motion and was scored from 0 to 10 during shoulder movement. High VAS scores indicate more severe pain.

\section{Maximal muscle power of the shoulder using a hand- held dynamometry}

An isometric resistance test was performed using a handheld dynamometer (MicroFET2; Biometrics Europe BV, Almere, The Netherlands) to assess the maximum force in 


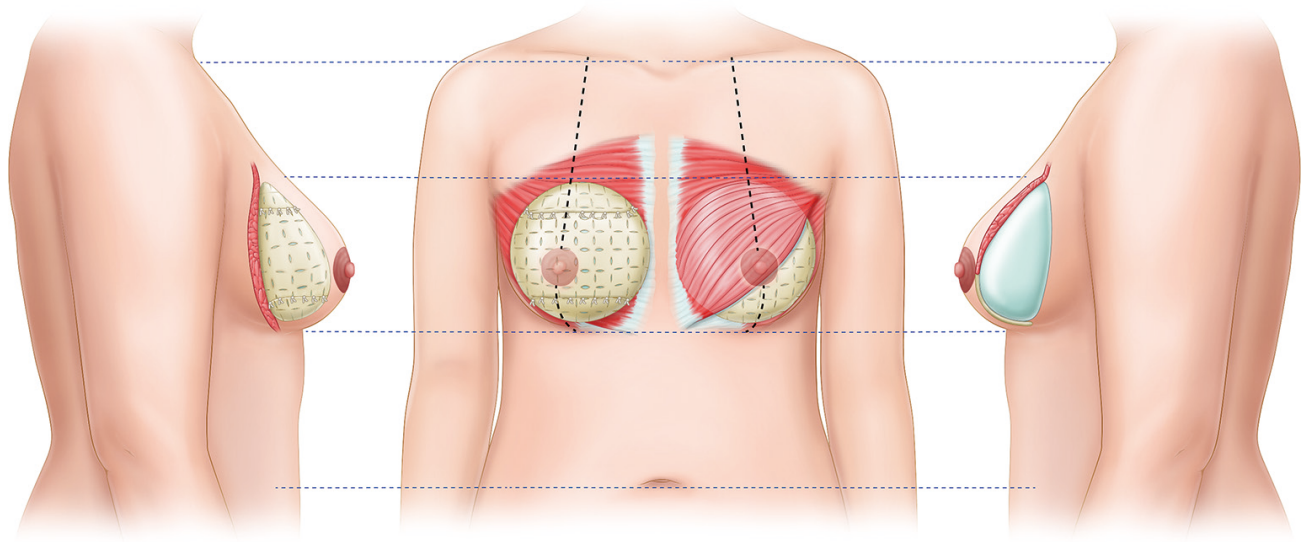

Figure 1 Concept illustration of the surgical techniques. The right breast shows the prepectoral technique in the experimental group. The full coverage technique used in this study is the double-crossed ADM technique, which preserves the pectoralis major muscle. The left breast shows the dual plane technique, which is currently the most utilized subpectoral technique. This technique makes an implant pocket using ADM in the region where the pectoralis major muscle of the infero-lateral part is lacking. ADM, acellular dermal matrix.
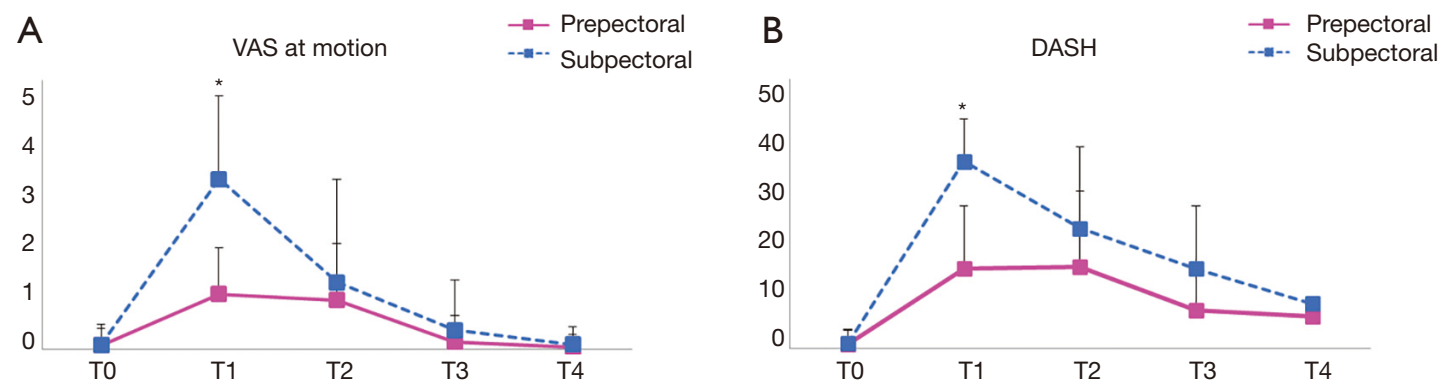

Figure 2 Statistical analysis: the intensity of pain and disability of the upper extremity. (A) There were significant differences in time and VAS score interaction effects between the two groups $(\mathrm{F}=4.317, \mathrm{P}=0.034)$. The prepectoral group exhibited lower VAS scores than the subpectoral group at 2 weeks post-operation, $\mathrm{T} 1(\mathrm{t}=-4.662, \mathrm{P}=0.000)$. (B) There were significant differences in time and DASH score interaction effects between the two groups $(\mathrm{F}=5.191, \mathrm{P}=0.013)$. VAS, visual analog scale; DASH, Disabilities of the Arm, Shoulder and Hand.

the six main directions of the ipsilesional shoulder in the supine position (12). The dynamometer was placed just proximal to the epicondyles of the humerus for the forward flexion, extension, abduction, and adduction. For internal rotation and external rotation, the dynamometer was placed just proximal to the ulnar and radial styloid processes during the $90^{\circ}$ forward flexion of the shoulder and $90^{\circ}$ flexion of the elbow. The participants were instructed to exert a maximal effort against the dynamometer and to optimally relax the other parts of the body during the tests. A physiatrist tested three trials in every direction. Each trial consisted of a muscle contraction of 5 seconds, and there was a relaxation period of at least 30 seconds between successive trials.

\section{Disability of the upper extremity}

The disabilities of the shoulder function were assessed using the Disabilities of the Arm, Shoulder and Hand (DASH) questionnaire, which consists of 30 items each with five responses (13); Twenty-one questionnaire items assess the degree of difficulty in performing different physical activities, six items assess symptoms, and the remaining three items assess psychosocial effects. A score of 0 indicates 'no disability' and a score of 100 indicates 'complete disability' (14). In this study, we used the Korean version of the questionnaire, and its reliability and validity for measuring the upper extremity dysfunction have been proven (13). 


\section{Quality of life using the 36-Item Short-Form Health} Survey

Health-related QOL has been used extensively in clinical and epidemiologic research and health service studies. The 36-Item Short-Form Health Survey (SF-36) is a widely used, generic, and patient-reported measure of health status (15). It comprises of four physical domain scales: physical functioning, physical role functioning, bodily pain, and general health. These four components are combined in a physical component summary scale. The survey also comprises four mental domain scales: vitality, social functioning, emotional role functioning, and mental health, which are combined in a mental component summary scale. We used the Korean version of the SF-36, and its reliability and validity have been proven (16).

\section{Anxiety and depressive mood status}

We assessed patients' mood status at each time point using the hospital anxiety and depression scale (HADS). In 1983, Zigmond et al. (17) developed a 14-question scale to measure the level of anxiety and depression in patients who visited the general hospitals. Seven odd items are used to measure anxiety, and seven even items are used to measure depression. Each item is scored from 0-3 points, and the total score is 21 points for anxiety and depression. When anxiety and depression items are scored as 8 points, they showed high sensitivity and specificity. Oh et al. (18) had the scale translated into Korean and performed standardization studies in Korea.

\section{Statistical analysis}

All statistical analyses were performed using IBM SPSS Statistics for Windows, version 23.0 (IBM Corp., Armonk, NY, USA). Assessment data were determined to be normally distributed according to a Shapiro-Wilk test. The clinical characteristics of participants and differences in the initial distribution of the range of motion, maximal muscle power, VAS score, DASH score, SF-36 score, and HADS score between the two groups were analyzed using a chi-squared test or $t$-test. Repeated-measures analysis of variance (RM-ANOVA) was performed to assess changes in functional outcome with five time points as the withingroup factor and two implant groups as the between-group factor. A Bonferroni multiple comparisons for correction was performed between the two implant groups at each time point $(\mathrm{P}<0.01)$.

\section{Results}

Between February 2018 and September 2019, DTI breast reconstruction was performed using a classical subpectoral technique in 14 patients and a prepectoral breast reconstruction using the double-crossed ADM coverage technique in 20 patients (Table 1). Patients' data in the subpectoral and prepectoral groups were as follows: mean age, 45.5/45.1 years (range, $44-56$ years; $P$ value, 0.787 ); BMI, $22.6 / 22.7 \mathrm{~kg} / \mathrm{m}^{2}$ (range, $18.6-25.2 \mathrm{~kg} / \mathrm{m}^{2}$; $\mathrm{P}$ value, 0.359); preoperative breast volume, 244.5/ $206.0 \mathrm{~cm}^{3}$ (range, $150-580 \mathrm{~cm}^{3}$; P value, 0.140 ); excised mass weight, $225.11 / 238.9 \mathrm{~g}$ (range, $110-477 \mathrm{~g}$; P value, 0.918 ); silicone implant volume, 258.6/234.8 $\mathrm{cm}^{3}$ (range, $125-440 \mathrm{~cm}^{3}$; P value, 0.800 ). All patients were nonsmokers and no patient had diabetes mellitus. Skin-sparing or nipple-sparing mastectomies were performed in all patients. There were no patients who received preoperative radiotherapy and chemotherapy. Adjuvant radiotherapy and chemotherapy were performed in $5 / 5$ and $5 / 6$ patients in the subpectoral and prepectoral group, respectively, and adjuvant therapy was performed in 12/19 patients (Table 1 ).

There were no significant differences in the distribution of age, tumor location, cancer stage, dissection, lymph node dissection, and presence of adjuvant chemotherapy, radiotherapy, and hormone therapy between the two groups (Table 1). Moreover, there were no significant differences in the initial distribution of the range of motion, maximal muscle power, VAS score, DASH score, SF-36 score, and HADS score between the two groups at T0 (Table 2).

There were significant differences in the time and VAS score interaction effects between the two groups $(\mathrm{F}=4.317, \mathrm{P}$ $=0.034$ ) (Figure $2 A$ ). In the post-hoc analysis, the prepectoral group exhibited lower VAS scores than the subpectoral group at 2 weeks post-operation, $\mathrm{T} 1(\mathrm{t}=-4.662, \mathrm{P}=0.000)$. Additionally, there were significant differences in the time and DASH score interaction effects between the two groups $(\mathrm{F}=5.191, \mathrm{P}=0.013)$ (Figure $2 B)$. In the post-hoc analysis, the prepectoral group exhibited lower DASH scores than that in the subpectoral group at $\mathrm{T} 1(\mathrm{t}=-4.825, \mathrm{P}=0.000)$. However, there were no significant differences in the time and maximal muscle force, and SF-36 and HADS scored interaction effects between the two groups (Tables 3-5).

\section{Discussion}

We analyzed the functional recovery of the patient group treated with the subpectoral technique and the 
Table 1 Clinical characteristics of participants

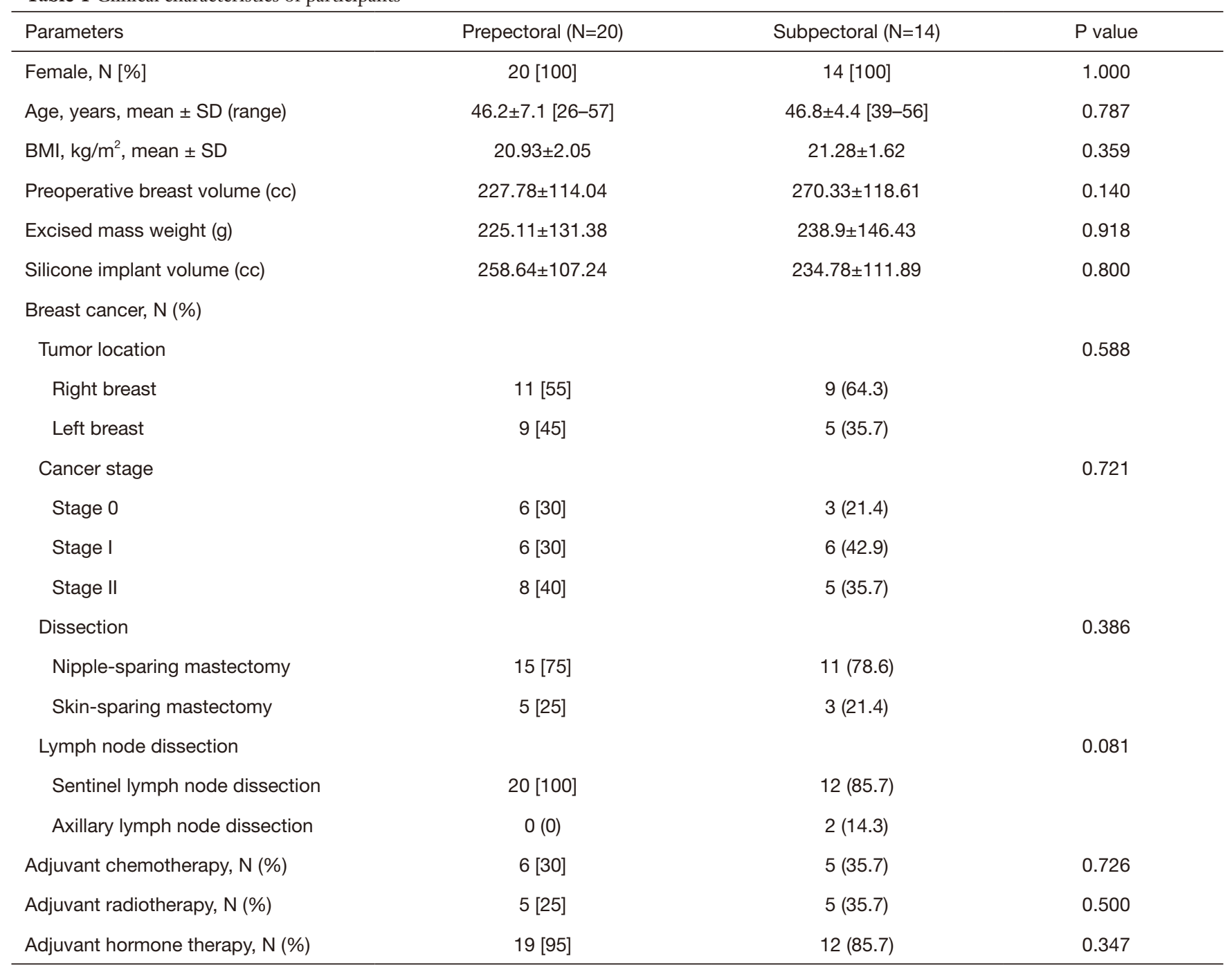

$\mathrm{N}$, the number of patients; mean $\pm \mathrm{SD}$, mean \pm standard deviation.

patient group treated with a full wrapping prepectoral technique using crossed-double ADM (Figure 1). Nelson et al. (19) summarized the functional impact after breast reconstruction and mentioned various objective test methods and the importance of postoperative rehabilitation. In this regard, this present study conducted research in cooperation with the Department of Rehabilitation Medicine to confirm a more objective and accurate functional recovery. Preoperational tests on pain, disability, maximal muscle power, the QOL questionnaire, hospital anxiety, and the depression scale were carried out to confirm that there were no statistically significant differences between the prepectoral and subpectoral group prior to the observations
(Table 2). Interestingly, there was statistically significant difference when the pain score during shoulder motion was compared, the prepectoral group showed a statistically significant decrease in pain at 2 weeks post-operation $(\mathrm{P}=0.034)$. In the DASH, which indicates disability, the prepectoral group showed significantly better scores than the subpectoral group at 2 weeks post-operation (Figure 2). Furthermore, no statistically significant difference was found between the prepectoral and subpectoral groups. However, when the recovery flow was examined by a breast reconstruction surgeon, there was early functional recovery (the focus of this study), which started at the original state at 2 weeks post-operation; and continued after 3 months, 
Table 2 The Initial distribution of the functional outcome at pre-operation (T0)

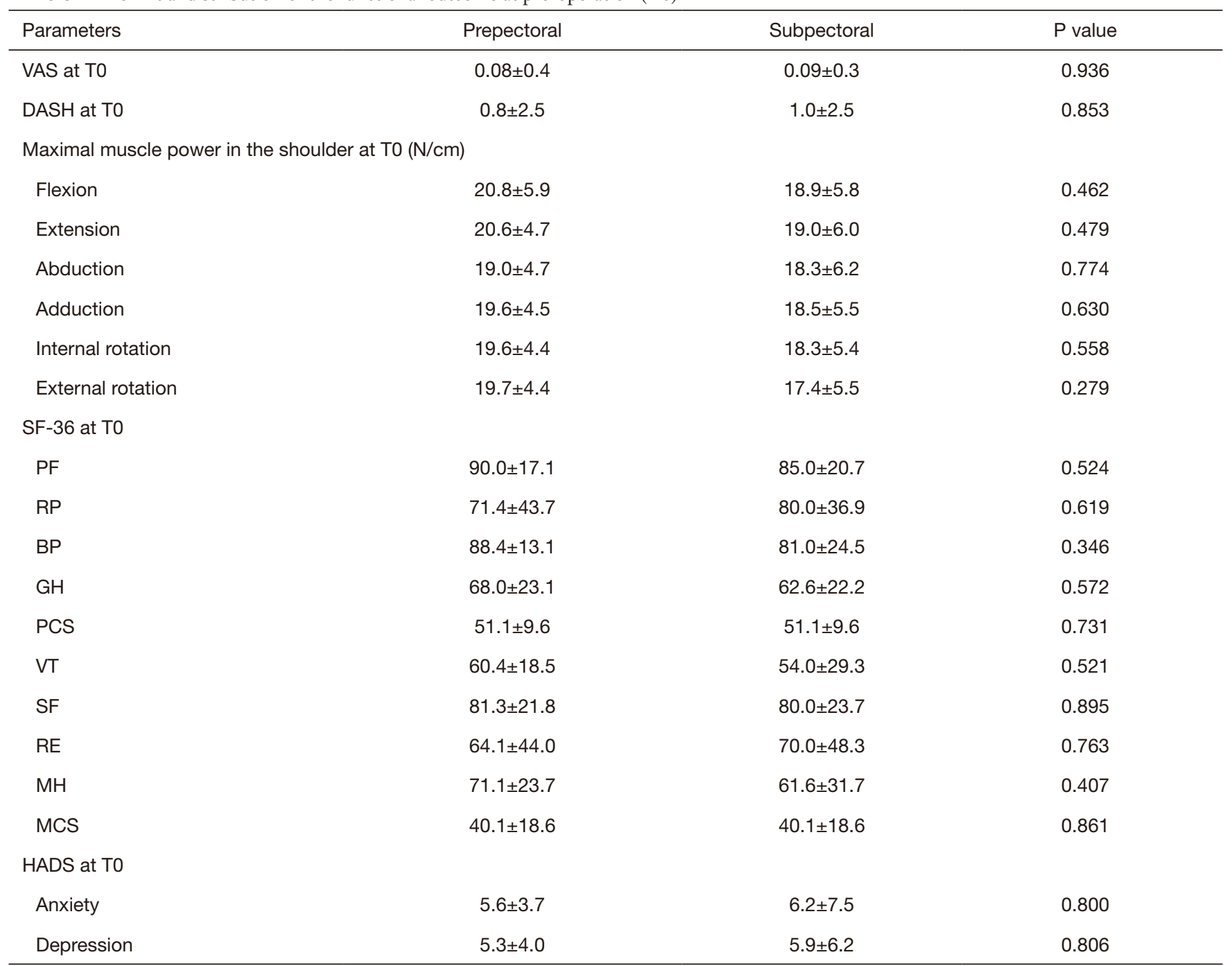

Each cell represents mean \pm standard deviation. VAS, visual analog scale; DASH, disabilities of the arm, shoulder and hand; SF-36, 36-Item Short-Form Health Survey; HADS, hospital anxiety and depression scale; PF, physical functioning; RP, physical role functioning; BP, bodily pain; GH, general health; PCS, physical component summary; VT, vitality; SF, social functioning; RE, emotional role functioning; $\mathrm{MH}$, mental health; MCS, mental component summary; T0, pre-operation.

and nearly all groups showed recovery. Specifically, each item recovered to the preoperative value at 6 months postoperation (Table 3). This aspect shows a similar flow when the SF-36 (representing the QOL) was checked, and the difference between the preoperative value and the worst 2 weeks post-operation value in the prepectoral group was insignificant. Reflecting this trend, there was a higher variation in the anxiety and depression of the subpectoral group at the 2 weeks post-operation; remarkably, at the last follow-up at 6 months, the prepectoral group showed a greater value than the preoperative group (Table 5). This might be a result of the overlapping concerns regarding surgical treatment after an increase in anxiety and depression from the diagnosis of breast cancer.

The prepectoral and subpectoral techniques in DTI breast reconstruction are difficult to divide evenly between patients. Although there are several good reports on the prepectoral technique, since the implant is located just below the mastectomy flap, it is important to set up careful indications before and after surgery in the patient selection. If there is a thin skin flap that is more likely to cause complications or low blood supply, or if there is an 
Table 3 The value of maximal muscle power of the shoulder

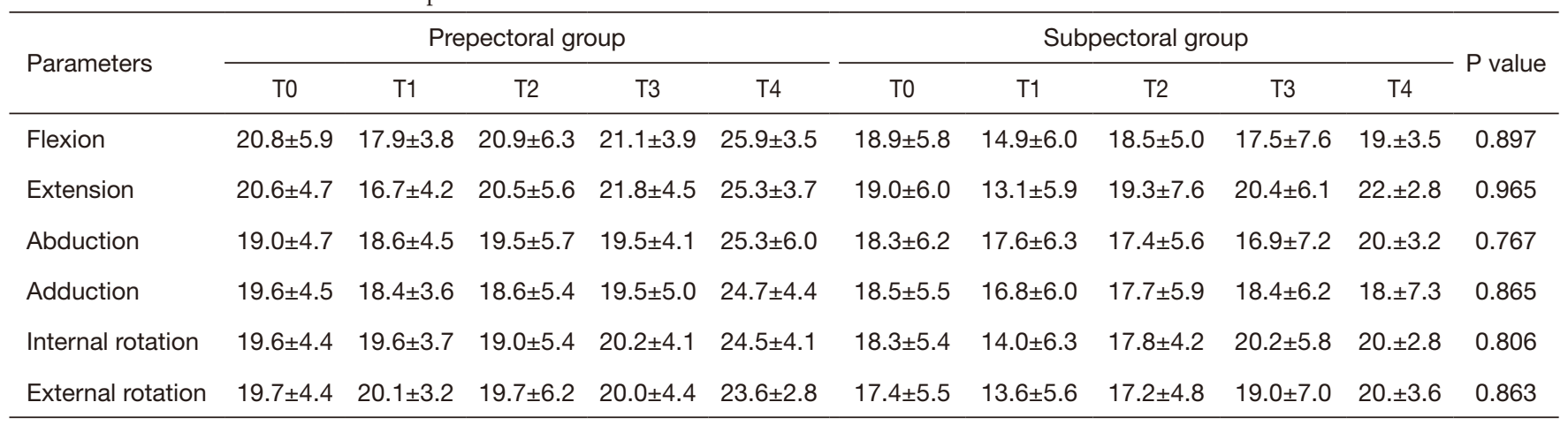

Each cell represents mean \pm standard deviation $(\mathrm{N} / \mathrm{cm})$. T0: pre-operation; T1: 2 weeks after operation; T2: 1 month after operation; T3: 3 months after operation; T4: 6 months after operation.

Table 4 The value of the 36-Item Short-Form Health Survey

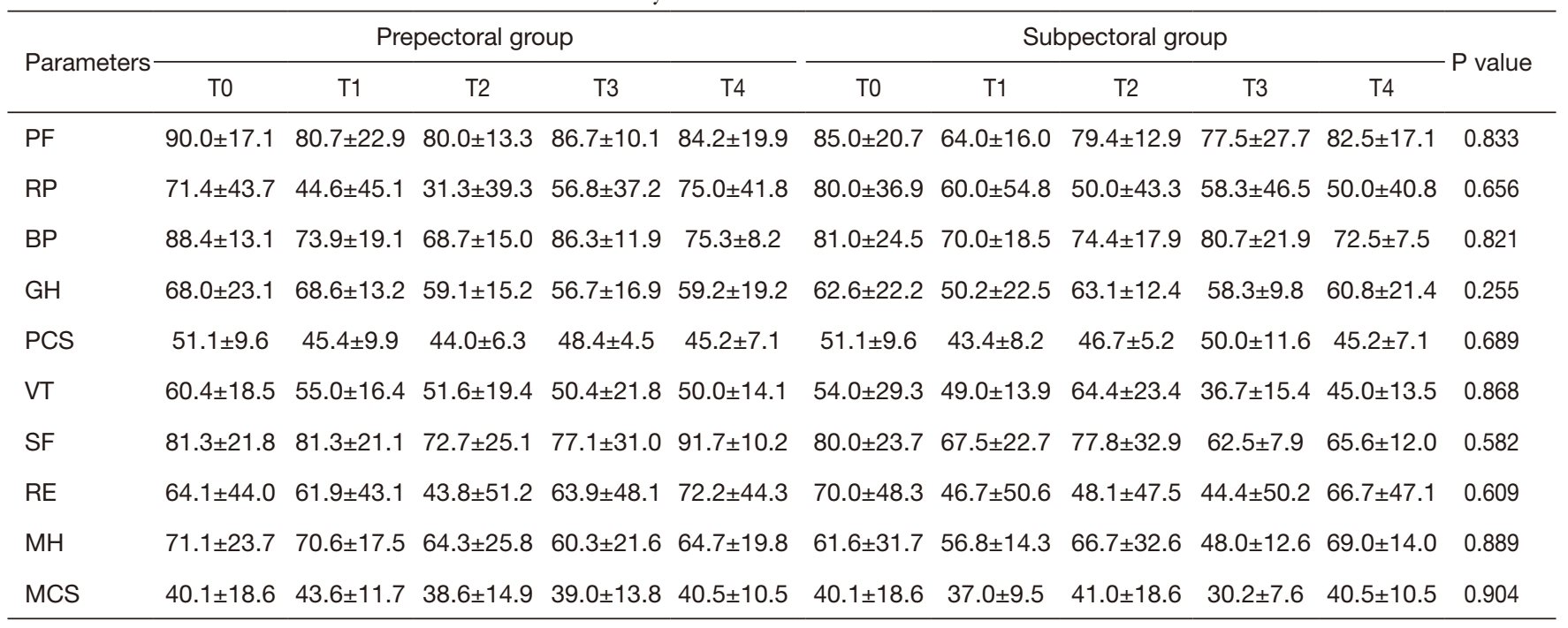

Each cell represents mean \pm standard deviation. PF, physical functioning; RP, physical role functioning; BP, bodily pain; GH, general health; PCS, physical component summary; VT, vitality; SF, social functioning; RE, emotional role functioning; $\mathrm{MH}$, mental health; MCS, mental component summary; T0, pre-operation; T1, 2 weeks after operation; T2, 1 month after operation; T3, 3 months after operation; T4, 6 months after operation.

Table 5 The value of severity of anxiety and depression

\begin{tabular}{|c|c|c|c|c|c|c|c|c|c|c|c|}
\hline Parameters & \multicolumn{5}{|c|}{ Prepectoral group } & \multicolumn{5}{|c|}{ Subpectoral group } & $P$ value \\
\hline Anxiety & $5.6 \pm 3.7$ & $5.1 \pm 3.8$ & $5.4 \pm 4.9$ & $6.8 \pm 5.8$ & $6.3 \pm 3.3$ & $6.2 \pm 7.5$ & $4.6 \pm 1.7$ & $6.1 \pm 4.2$ & $4.8 \pm 1.9$ & $5.0 \pm 2.9$ & 0.959 \\
\hline Depression & $5.3 \pm 4.0$ & $5.1 \pm 3.2$ & $5.4 \pm 4.3$ & $6.4 \pm 4.4$ & $7.5 \pm 7.2$ & $5.9 \pm 6.2$ & $4.8 \pm 2.3$ & $5.8 \pm 4.1$ & $5.8 \pm 4.0$ & $6.3 \pm 3.8$ & 0.924 \\
\hline
\end{tabular}

Each cell represents mean \pm standard deviation. T0, pre-operation; T1, 2 weeks after operation; T2, 1 month after operation; T3, 3 months after operation; T4, 6 months after operation. 
Table 6 Postoperative complications at T3 and T4

\begin{tabular}{lcc}
\hline Complication & Prepectoral group $(\mathrm{n}=20)$ & Subpectoral group $(\mathrm{n}=14)$ \\
\hline Seroma & $3(15 \%)$ & $2(14.3 \%)$ \\
Nipple sloughing/necrosis & $1 / 0(5 \% /-)$ & $2 / 0(14.3 \% /-)$ \\
Capsular contracture & $1(5 \%)$ & - \\
Infection/implant loss & - & - \\
Animation deformity & - & $2(14.3 \%)$ \\
Rippling & $1(5 \%)$ & $1(5 \%)$ \\
\hline
\end{tabular}

Each cell represents the number of patients. T3, 3 months after operation; T4, 6 months after operation.

underlying disease that can slow wound healing, it is a contradiction of the prepectoral technique. In addition, rippling is more likely to occur in the prepectoral groups, while animation deformity is more likely in the subpectoral groups, given the basic principles of the surgical method. In this study, one patient $(5 \%)$ had capsular contracture (Backer stage I) in the prepectoral group, and two patients (14.3\%) had animation deformity in the subpectoral group, but they healed without major complications (Table 6).

If this aspect is comprehensively considered and applied to the appropriate patient to minimize the potential postsurgical complications, the patient's pain, disability, and anxiety can be lowered, and QOL can be increased following improved patient satisfaction. In addition, as confirmed in this study, the prepectoral group with a preserved pectoralis major muscle showed a slightly better outcome until the second week post-operation; however, 3 and 6 months after the surgery, both techniques showed recovery to the preoperative state (Tables 3-5). This study is meaningful for its prospective comparison, but both groups had small sample size. The prepectoral technique is also expensive because it requires a larger area $\mathrm{ADM}$ for full coverage of the implant. Therefore, this study is considered suitable as a preliminary report to analyze the functional outcome in the large patient group in the future, and we are preparing to continue this study of patient-specific surgery for more patients.

Furthermore, in recent years, as the implementation of prophylactic mastectomy due to the $B R C A$ gene testing is increasing and the patient group's age is decreasing, functional recovery and a fast return to daily life are being prioritized. For this reason, the need for carrying out breast reconstruction without scarifying the anatomical structure of the donor site using the self-tissue is increasing. In future, while choosing DTI breast reconstruction technique, surgeons should first consider oncological safety, and then think about how to reduce the complications and increase the QOL and patient satisfaction.

\section{Conclusions}

This study compared early functional outcome of the subpectoral plane and the prepectoral plane of DTI breast reconstruction. Pain and disability two weeks post-operation were statistically significant in the prepectoral technique, which preserves the pectoralis major muscle. However, this study predicts that better outcomes can be expected if patient selection is optimized. This study, additionally, sets a platform for larger studies in future.

\section{Acknowledgments}

Funding: None.

\section{Footnote}

Reporting Checklist: The authors have completed the STROBE reporting checklist. Available at http://dx.doi. org/10.21037/apm-20-1550

Data Sharing Statement: Available at http://dx.doi. org/10.21037/apm-20-1550

Conflicts of Interest: All authors have completed the ICMJE uniform disclosure form (available at http://dx.doi. org/10.21037/apm-20-1550). The authors have no conflicts of interest to declare.

Ethical Statement: The authors are accountable for all aspects of the work in ensuring that questions related to the accuracy or integrity of any part of the work 
are appropriately investigated and resolved. The study was conducted in accordance with the Declaration of Helsinki (as revised in 2013). The study was approved by the Institutional Review Board of Kyungpook National University Chilgok Hospital (No. 2018-05-005-001). Informed consent was obtained from all individual participants included in the study.

Open Access Statement: This is an Open Access article distributed in accordance with the Creative Commons Attribution-NonCommercial-NoDerivs 4.0 International License (CC BY-NC-ND 4.0), which permits the noncommercial replication and distribution of the article with the strict proviso that no changes or edits are made and the original work is properly cited (including links to both the formal publication through the relevant DOI and the license). See: https://creativecommons.org/licenses/by-nc-nd/4.0/.

\section{References}

1. Stubblefield MD, Custodio CM. Upper-extremity pain disorders in breast cancer. Arch Phys Med Rehabil 2006;87:S96-9.

2. Shamley D, Lascurain-Aguirrebeña I, Oskrochi R, et al. Shoulder morbidity after treatment for breast cancer is bilateral and greater after mastectomy. Acta Oncol 2012;51:1045-53.

3. de Haan A, Toor A, Hage JJ, et al. Function of the pectoralis major muscle after combined skin-sparing mastectomy and immediate reconstruction by subpectoral implantation of a prosthesis. Ann Plast Surg 2007;59:605-10.

4. Reitsamer R, Peintinger F. Prepectoral implant placement and complete coverage with porcine acellular dermal matrix: A new technique for direct-to-implant breast reconstruction after nipple-sparing mastectomy. J Plast Reconstr Aesthet Surg 2015;68:162-7.

5. Downs RK, Hedges K. An alternative technique for immediate direct-to-implant breast reconstruction: A case series. Plast Reconstr Surg Glob Open 2016;4:e821.

6. Lee JS, Kim JS, Lee JH, et al. Prepectoral breast reconstruction with complete implant coverage using double-crossed acellular dermal matrixs. Gland Surg 2019;8:748-57.

7. Ibrahim AM, Koolen PG, Ashraf AA, et al. Acellular dermal matrix in reconstructive breast surgery: Survey of current practice among plastic surgeons. Plast Reconstr Surg Glob Open 2015;3:e381.

8. Gandhi A, Barr L, Johnson R. Bioprosthetics: Changing the landscape for breast reconstruction? Eur J Surg Oncol 2013;39:24-5.

9. Macadam SA, Lennox PA. Acellular dermal matrices: Use in reconstructive and aesthetic breast surgery. Can J Plast Surg 2012;20:75-89.

10. Schaeffer CV, Dassoulas KR, Thuman J, et al. Early functional outcomes after prepectoral breast reconstruction: A case-matched cohort study. Ann Plast Surg 2019;82:S399-S403.

11. Alba B, Schultz B, Qin LA, et al. Postoperative upper extremity function in implant and autologous breast reconstruction. J Reconstr Microsurg 2020;36:151-6.

12. Hage JJ, van der Heeden JF, Lankhorst KM, et al. Impact of combined skin sparing mastectomy and immediate subpectoral prosthetic reconstruction on the pectoralis major muscle function: a preoperative and postoperative comparative study. Ann Plast Surg 2014;72:631-7.

13. Lee JY, Lim JY, Oh JH, et al. Cross-cultural adaptation and clinical evaluation of a Korean version of the disabilities of arm, shoulder, and hand outcome questionnaire (K-DASH). J Shoulder Elbow Surg 2008;17:570-4.

14. Glassey N, Perks GB, McCulley SJ. A prospective assessment of shoulder morbidity and recovery time scales following latissimus dorsi breast reconstruction. Plast Reconstr Surg 2008;122:1334-40.

15. Ware Jr JE, Sherbourne CD. The MOS 36-item short-form health survey (SF-36): I. Conceptual framework and item selection. Med Care 1992;30:473-83.

16. Han CW, Lee EJ, Iwaya T, et al. Development of the Korean version of Short-Form 36-Item Health Survey: health related QOL of healthy elderly people and elderly patients in Korea. Tohoku J Exp Med 2004;203:189-94.

17. Zigmond AS, Snaith RP. The hospital anxiety and depression scale. Acta Psychiatr Scand 1983;67:361-70.

18. Oh SM, Min KJ, Park DB. A study on the standardization of the hospital anxiety and depression scale for Koreans: a comparison of normal, depressed and anxious groups. J Korean Neuropsychiatr Assoc 1999;38:289.

19. Nelson JA, Lee IT, Disa JJ. The functional impact of breast reconstruction: An overview and update. Plast Reconstr Surg Glob Open 2018;6:e1640.

Cite this article as: Lee JS, Park E, Lee JH, Lee J, Park HY, Yang JD, Jung TD. A prospective comparison study of early functional outcomes after implant-based breast reconstruction: subpectoral versus prepectoral technique. Ann Palliat Med 2021;10(3):2520-2529. doi: 10.21037/apm-20-1550 